\title{
On holomorphic isometric embeddings of the unit $n$-ball into products of two unit $m$-balls
}

\author{
Sui-Chung, $\mathrm{Ng}^{*}$
}

\begin{abstract}
We study holomorphic isometric embeddings of the complex unit $n$-ball into products of two complex unit $m$-balls with respect to their Bergman metrics up to normalization constants (the isometric constant). There are two trivial holomorphic isometric embeddings for $m \geq n$, given by $F_{1}(\mathbf{z})=\left(\mathbf{0}, I_{n ; m}(\mathbf{z})\right)$ with the isometric constant equal to $(m+1) /(n+1)$ and $F_{2}(\mathbf{z})=$ $\left(I_{n ; m}(\mathbf{z}), I_{n ; m}(\mathbf{z})\right)$ with the isometric constant equal to $2(m+1) /(n+1)$. Here $I_{n ; m}: \mathbb{C}^{n} \longrightarrow \mathbb{C}^{m}$ is the canonical embedding. We prove that when $m<2 n$, these are the only holomorphic isometric embeddings up to unitary transformations.
\end{abstract}

Mathematics Subject Classification (2000): 53C35, 53C55

Keywords: Holomorphic isometric embeddings, complex unit balls, Bergman metrics, total geodesy

\section{Introduction}

Let $\Omega$ be an irreducible bounded symmetric domain equipped with its Bergman metric $d s_{\Omega}^{2}$. In relation to a problem in number theory, Clozel and Ullmo [1] studied the holomorphic isometric embeddings of $\Omega$ into its Cartesian products $\Omega^{p}$ up to normalizing constants, in which $\Omega^{p}$ is equipped with the product metric. By using the arguments in Hermitian metric rigidity (see Mok $[2,3]$ ), they argued in their article that when $\operatorname{rank}(\Omega) \geq 2$, any such embedding must be totally geodesic. When $\operatorname{rank}(\Omega)=1$, i.e. when $\Omega=\mathbb{B}^{n}$, the complex unit balls, Mok [4] showed that for $n \geq 2$, the embeddings must also be totally geodesic. While for dimension 1 , he has constructed a non-totally geodesic holomorphic isometric embedding of the unit disk $\Delta$ into $\Delta^{p}$ for every $p \geq 2$. (see [5])

Let $m, n \geq 2$ be two integers. In this article, we consider holomorphic isometric embeddings of $\mathbb{B}^{n}$ into $\mathbb{B}^{m} \times \mathbb{B}^{m}$ up to normalization constants with respect to their Bergman metrics $d s_{\mathbb{B}^{n}}^{2}$ and $d s_{\mathbb{B}^{m} \times \mathbb{B}^{m}}^{2}$. More precisely, for a positive real number $\lambda, F: \mathbb{B}^{n} \longrightarrow \mathbb{B}^{m} \times \mathbb{B}^{m}$ is said to be a holomorphic isometric embedding with the isometric constant $\lambda$ if $F:\left(\mathbb{B}^{n}, \lambda d s_{\mathbb{B}^{n}}^{2}\right) \longrightarrow$ $\left(\mathbb{B}^{m} \times \mathbb{B}^{m}, d s_{\mathbb{B}^{m} \times \mathbb{B}^{m}}^{2}\right)$ is a holomorphic isometric embedding. If $m \geq n$ and $I_{n ; m}: \mathbb{C}^{n} \longrightarrow \mathbb{C}^{m}$ is the canonical embedding, then $F_{1}(\mathbf{z})=\left(\mathbf{0}, I_{n ; m}(\mathbf{z})\right)$ and $F_{2}(\mathbf{z})=\left(I_{n ; m}(\mathbf{z}), I_{n ; m}(\mathbf{z})\right)$ are two holomorphic isometric embeddings with the isometric constant equal to $(m+1) /(n+1)$ and

*Address: Department of Mathematics, The University of Hong Kong, Pokfulam Road, Hong Kong. Email: math.scng@hku.hk 
$2(m+1) /(n+1)$ respectively. The main purpose of this paper is to prove that for $m<2 n$, they are the only holomorphic isometric embeddings up to unitary transformations.

Main theorem Let $m, n$ be positive integers with $m, n \geq 2$ and $m<2 n$. Let $F: \mathbb{B}^{n} \longrightarrow$ $\mathbb{B}^{m} \times \mathbb{B}^{m}$ be a holomorphic isometric embedding with the isometric constant $\lambda$. Then $m \geq n$ and up to unitary transformations, either $F(\mathbf{z})=\left(\mathbf{0}, I_{n ; m}(\mathbf{z})\right)$ with $\lambda=(m+1) /(n+1)$, or $F(\mathbf{z})=\left(I_{n ; m}(\mathbf{z}), I_{n ; m}(\mathbf{z})\right)$ with $\lambda=2(m+1) /(n+1)$, where $I_{n ; m}: \mathbb{C}^{n} \longrightarrow \mathbb{C}^{m}$ is the canonical embedding.

\section{Functional equation}

Let $m, n \geq 2$ be two integers and $F: \mathbb{B}^{n} \longrightarrow \mathbb{B}^{m} \times \mathbb{B}^{m}, F(\mathbf{z})=(A(\mathbf{z}), B(\mathbf{z}))$ be a holomorphic isometric embedding with the isometric constant $\lambda$. Without loss of generality, we may assume that $F(\mathbf{0})=(\mathbf{0}, \mathbf{0})$. The Bergman metric on $\mathbb{B}^{n}$ is given by $d s_{\mathbb{B}^{n}}^{2}=2 \operatorname{Re} \sum g_{i j} d z^{i} \otimes d \bar{z}^{j}$, where $g_{i \bar{j}}=-(n+1) \frac{\partial^{2}}{\partial z_{i} \partial \bar{z}_{j}} \log \left(1-\|\mathbf{z}\|^{2}\right)$. We write $\left(\mathbf{z}_{1}, \mathbf{z}_{2}\right)$ for a point in $\mathbb{B}^{m} \times \mathbb{B}^{m}$. We can take as Kähler potentials for $d s_{\mathbb{B}^{n}}^{2}$ and $d s_{\mathbb{B}^{m} \times \mathbb{B}^{m}}^{2}$ the real analytic functions $-(n+1) \log \left(1-\|\mathbf{z}\|^{2}\right)$ and $-(m+1) \log \left(1-\left\|\mathbf{z}_{1}\right\|^{2}\right)\left(1-\left\|\mathbf{z}_{2}\right\|^{2}\right)$ respectively. By the assumption that $F^{*} d s_{\mathbb{B}^{m} \times \mathbb{B}^{m}}^{2}=\lambda d s_{\mathbb{B}^{n}}^{2}$ it follows that

$$
-(m+1) \sqrt{-1} \partial \bar{\partial} \log \left(1-\|A\|^{2}\right)\left(1-\|B\|^{2}\right)=-\lambda(n+1) \sqrt{-1} \partial \bar{\partial} \log \left(1-\|\mathbf{z}\|^{2}\right),
$$

hence,

$$
(m+1) \log \left(1-\|A\|^{2}\right)\left(1-\|B\|^{2}\right)=\lambda(n+1) \log \left(1-\|\mathbf{z}\|^{2}\right)+\operatorname{Re} h
$$

for some holomorphic function $h$ on $\mathbb{B}^{n}$. Since $F(\mathbf{0})=(\mathbf{0}, \mathbf{0})$, by comparing Taylor expansions we conclude that $h \equiv 0$. Therefore we obtain

$$
(m+1) \log \left(1-\|A\|^{2}\right)\left(1-\|B\|^{2}\right)=\lambda(n+1) \log \left(1-\|\mathbf{z}\|^{2}\right) .
$$

i.e.

$$
\left(1-\|A\|^{2}\right)\left(1-\|B\|^{2}\right)=\left(1-\|\mathbf{z}\|^{2}\right)^{\lambda(n+1) /(m+1)} .
$$

Eq.(2.2) is a real-analytic equation and we can consider an associated polarized functional equation. In general, given two power series $\sum a_{i \bar{j}} z^{i} \bar{z}^{j}$ and $\sum b_{i \bar{j}} z^{i} \bar{z}^{j}$, they are equal if and only if $a_{i \bar{j}}=b_{i \bar{j}}, \forall i, j$. Therefore their equality will also imply the polarized equation $\sum a_{i \bar{j}} z^{i} \bar{w}^{j}=$ $\sum b_{i j} z^{i} \bar{w}^{j}$. Since we can polarize each variable separately, the polarized equation of Eq.(2.1) is

$$
(m+1) \log (1-\langle A(\mathbf{z}), A(\mathbf{w})\rangle)(1-\langle B(\mathbf{z}), B(\mathbf{w})\rangle)=\lambda(n+1) \log (1-\langle\mathbf{z}, \mathbf{w}\rangle)
$$

for $\|\mathbf{z}\|,\|\mathbf{w}\|<1$. Here $\log$ denotes the principal branch of the logarithm and $\langle$,$\rangle is the$ complex Euclidean inner product. We can rewrite it as

$$
(1-\langle A(\mathbf{z}), A(\mathbf{w})\rangle)(1-\langle B(\mathbf{z}), B(\mathbf{w})\rangle)=(1-\langle\mathbf{z}, \mathbf{w}\rangle)^{\lambda(n+1) /(m+1)},
$$

where

$$
(1-\langle\mathbf{z}, \mathbf{w}\rangle)^{\lambda(n+1) /(m+1)} \equiv e^{[\lambda(n+1) /(m+1)] \log (1-\langle\mathbf{z}, \mathbf{w}\rangle)} .
$$




\section{Algebraic extension}

In [5], Mok has established the following extension result.

Theorem 3.1 (Mok). Let $\Omega \Subset \mathbb{C}^{n}$ and $\Omega^{\prime} \Subset \mathbb{C}^{N}$ be bounded symmetric domains in their Harish-Chandra realizations. Let $\lambda$ be any positive real number and $f:\left(\Omega, \lambda d s_{\Omega}^{2}\right) \longrightarrow$ $\left(\Omega^{\prime}, d s_{\Omega^{\prime}}^{2}\right)$ be a germ of holomorphic isometry at $0 \in \Omega$ with $f(0)=0$. Then, the germ of the graph of $f$ extends to an affine algebraic variety $S^{\#} \subset \mathbb{C}^{n} \times \mathbb{C}^{N}$ such that $S=S^{\#} \cap\left(\Omega \times \Omega^{\prime}\right)$ is the graph of a holomorphic isometric embedding $F: \Omega \longrightarrow \Omega^{\prime}$ extending the germ of the holomorphic map $f$.

From the existence of algebraic extension, we can prove

Proposition 3.2. Let $\left(\mathbb{B}^{n}, \lambda d s_{\Delta}^{2}\right) \longrightarrow\left(\mathbb{B}^{m} \times \mathbb{B}^{m}, d s_{\mathbb{B}^{m} \times \mathbb{B}^{m}}^{2}\right)$ be a holomorphic isometric embedding. Then $\frac{\lambda(n+1)}{(m+1)}$ is a positive integer.

Proof. By Theorem 3.1, we know that the embedding can be extended across a general point on the unit sphere $\partial \mathbb{B}^{n}$. Let $\mathbf{z}_{\mathbf{0}}$ be a point on $\partial \mathbb{B}^{n}$ at which the embedding can be extended across in a neighborhood. By unitary transformations, we may assume that $\mathbf{z}_{\mathbf{0}}=\left(z_{0}, 0, \ldots, 0\right)$. Consider the restriction of $F$ on the disk $\Delta=\{(z, 0, \ldots, 0),|z|<1\} \subset \mathbb{B}^{n}$, denote by $f(z)=(a(z), b(z))$, where $a(z), b(z) \in \mathbb{B}^{m}$. Then by Eq.(2.3), $f(z)$ satisfies

$$
(1-\langle a(z), a(w)\rangle)(1-\langle b(z), b(w)\rangle)=(1-z \bar{w})^{\lambda(n+1) /(m+1)} .
$$

If we consider Eq.(3.1) and substitute $w=z_{0}$, then because each factor on the L.H.S. can only vanish with an integral order at $z=z_{0}$ and therefore $\frac{\lambda(n+1)}{(m+1)}$ on the R.H.S. must be a positive integer.

Write $k=\frac{\lambda(n+1)}{(m+1)}$. By Eq.(2.2) and Schwarz's lemma on holomorphic maps, we have $k \leq 2$ and hence $k=1,2$. When $k=2$, by Schwarz's lemma again, we must have $\|\mathbf{z}\|=$ $\|A\|=\|B\|$ and therefore $m \geq n$ and up to unitary transformations, $A(\mathbf{z})=B(\mathbf{z})=I_{n ; m}(\mathbf{z})$, where $I_{n ; m}: \mathbb{C}^{n} \longrightarrow \mathbb{C}^{m}$ is the canonical embedding. Thus, it remains to consider the case when $k=1$, i.e. $\lambda=(m+1) /(n+1)$.

We first state a well known lemma of holomorphic maps.

Lemma 3.3. Let $f: U \subset \mathbb{C}^{n} \longrightarrow \mathbb{C}^{m}, f=\left(f_{1}, \ldots, f_{n}\right)$ be a holomorphic map defined on some open set $U$ and write $\|f\|^{2}=\sum_{i=1}^{n}\left|f_{i}\right|^{2}$. If $g: U \longrightarrow \mathbb{C}^{m}$ is another holomorphic map with $\|f\|^{2}=\|g\|^{2}$, then there exists a unitary transformation $\mathbf{U}$ in $\mathbb{C}^{m}$ such that $\mathbf{U} \circ f=g$.

Let $F: \mathbb{B}^{n} \longrightarrow \mathbb{B}^{m} \times \mathbb{B}^{m}, F(\mathbf{z})=(A(\mathbf{z}), B(\mathbf{z}))$ be an isometric embedding with the isometric constant $\lambda=(m+1) /(n+1)$. Then the functional equation Eq.(2.2) satisfied by $F$ reduces to

$$
\left(1-\|A(\mathbf{z})\|^{2}\right)\left(1-\|B(\mathbf{z})\|^{2}\right)=1-\|\mathbf{z}\|^{2} .
$$

Proposition 3.4. Let $V$ be the irreducible $n$-dimensional algebraic subvariety in $\mathbb{C}^{n} \times\left(\mathbb{C}^{m}\right)^{2}$ extending the graph of $F$ and $\pi$ be the projection map from $V$ to the first factor. There exists a proper algebraic subvariety $W \subset \mathbb{C}^{n}$ such that the restriction $\pi: V \backslash \pi^{-1}(W) \longrightarrow \mathbb{C}^{n} \backslash W$ is a finite unbranched covering map. 
Proof. From Eq.(3.2),

$$
\begin{gathered}
\|A\|^{2}+\|B\|^{2}=\|A\|^{2}\|B\|^{2}+\|\mathbf{z}\|^{2} . \\
\Longleftrightarrow \sum_{i=1}^{m}\left|a_{i}\right|^{2}+\sum_{i=1}^{m}\left|b_{i}\right|^{2}=\sum_{i=1}^{m} \sum_{j=1}^{m}\left|a_{i} b_{j}\right|^{2}+\sum_{i=1}^{n}\left|z_{i}\right|^{2} .
\end{gathered}
$$

By Lemma 3.3, (because $m^{2}+n>2 m$ ) there exists an $\left(m^{2}+n\right) \times\left(m^{2}+n\right)$ unitary matrix U such that

$$
\left[\begin{array}{c}
a_{1} \\
\vdots \\
a_{m} \\
b_{1} \\
\vdots \\
b_{m} \\
0 \\
\vdots \\
0
\end{array}\right]=\mathbf{U}\left[\begin{array}{c}
a_{1} b_{1} \\
\vdots \\
a_{1} b_{m} \\
\vdots \\
a_{m} b_{1} \\
\vdots \\
a_{m} b_{m} \\
z_{1} \\
\vdots \\
z_{n}
\end{array}\right]
$$

Consider the first $2 m$ equations above, they are

$$
\begin{aligned}
a_{1}= & L_{1}^{a}\left(a_{1} b_{1}, \ldots, a_{m} b_{m}, z_{1}, \ldots, z_{n}\right) \\
\vdots & \vdots \\
a_{m}= & L_{m}^{a}\left(a_{1} b_{1}, \ldots, a_{m} b_{m}, z_{1}, \ldots, z_{n}\right) \\
b_{1}= & L_{1}^{b}\left(a_{1} b_{1}, \ldots, a_{m} b_{m}, z_{1}, \ldots, z_{n}\right) \\
\vdots & \vdots \\
b_{m}= & L_{m}^{b}\left(a_{1} b_{1}, \ldots, a_{m} b_{m}, z_{1}, \ldots, z_{n}\right)
\end{aligned}
$$

where $L_{i}^{a}, L_{j}^{b}$ are some linear functions.

By applying the Implicit Function Theorem, we see that the algebraic subvariety defined by these $2 m$ equations is smooth at the origin. Therefore $V$ is the irreducible component of this algebraic subvariety containing the origin. Let $\bar{V}$ be the closure of $V$ in $\mathbb{P}^{n} \times\left(\mathbb{P}^{m}\right)^{2} . \bar{V}$ is obtained by replacing the inhomogeneous coordinates of the algebraic equations defining $V$ by homogeneous coordinates and $\bar{V}$ is a proper analytic subvariety of $\mathbb{P}^{n} \times\left(\mathbb{P}^{m}\right)^{2}$.

The singular part of $\bar{V}$ is a proper analytic subvariety $S$ of $\bar{V}$. By Proper Mapping Theorem, $\pi(S)$ is a proper analytic subvariety of $\mathbb{P}^{n}$. Thus, when restricting on $\bar{V}^{\prime}=\bar{V} \backslash \pi^{-1}(\pi(S))$, $\pi$ is a proper holomorphic map between complex manifolds and let us denote by $R$ the ramification locus of $\pi$. Let $\bar{R}$ be the closure of $R$ in $\bar{V}$. We are going to show that $\bar{R}$ is a proper analytic subvariety of $\bar{V}$. Take a point $v \in \bar{R}$ and let $U$ be a small coordinate open ball in $\mathbb{P}^{n} \times\left(\mathbb{P}^{m}\right)^{2}$ containing $v$ such that $\bar{V}$ is defined by $h_{1}=\cdots=h_{2 m}=0$ for some holomorphic functions $h_{j}, 1 \leq j \leq 2 m$, in $U$. Let $\left(u_{1}, \ldots, u_{n+2 m}\right)$ be a coordinate system of $U$. Write $\pi=\left(p_{1}, \ldots, p_{n}\right)$, where $p_{i}$ are holomorphic in $U$. Then $R$ is defined by the equation $\left.d p_{1} \wedge \cdots \wedge d p_{n}\right|_{\bar{V}^{\prime}}=0$. Take $y$ be a point in $\bar{V}^{\prime} \backslash R$. By doing a linear change of coordinates, we may assume that $\frac{\partial}{\partial u_{j}}, 1 \leq j \leq n$ are tangent to $\bar{V}$ at the point $y$, and hence $\left(\frac{\partial p_{i}}{\partial u_{j}}\right)_{1 \leq i, j \leq n}$ is non-singular at $y$. 
Claim: There exist holomorphic functions $f_{1}, \ldots, f_{2 m}$ in $U$ such that for $1 \leq k \leq 2 m$, $\left.f_{k}\right|_{\bar{V}}=0$ and $d f_{k}(y)=d u_{n+k}(y)$.

Let us assume the claim for the moment. Denote by $\mathcal{R}$ the analytic subvariety of $U$ defined by $d p_{1} \wedge \cdots \wedge d p_{n} \wedge d f_{1} \wedge \cdots \wedge d f_{2 m}=0$. $\mathcal{R}$ is a proper subvariety because it does not contain $y$ by our construction. Let $\widetilde{R}=\mathcal{R} \cap \bar{V}$. $\widetilde{R}$ is then a subvariety in $\bar{V} \cap U$ of codimension 1 and $\bar{R} \cap U \subset \widetilde{R}$ by our construction. $\widetilde{R}$ has only a finite number of irreducible components and let $\widetilde{R}_{l}, 1 \leq l \leq q$ be those having non-empty intersections with $R \cap U$. Since both $R \cap U$ and $\widetilde{R}$ are divisors in $U$ and $(R \cap U) \subset \widetilde{R}$, we must have $\bar{R} \cap U=\bigcup_{l=1}^{q} \widetilde{R}_{l}$. Thus, $\bar{R}$ is an analytic subvariety of $\bar{V}$.

Now Proper Mapping Theorem says that $\pi(\bar{R})$ is an analytic subvariety of $\mathbb{P}^{n}$. If we let $\bar{W}=\pi(S) \cup \pi(\bar{R})$, then $\pi: \bar{V} \backslash \pi^{-1}(\bar{W}) \longrightarrow \mathbb{P}^{n} \backslash \bar{W}$ is a proper holomorphic covering map. It is finite because $\pi$ is proper and discrete on $\bar{V} \backslash \pi^{-1}(\bar{W})$. We can obtain the conclusion of the proposition by just restricting $\pi$ to the finite part of $\mathbb{P}^{n} \times\left(\mathbb{P}^{m}\right)^{2}$.

Proof of the claim: It is an extension problem with a prescribed first order derivative at $y$. We will use Cartan's Theorem B. Assume that the coordinates of $y$ are $u_{1}=\cdots=u_{n+2 m}=0$. Let $\mathcal{O}=\mathcal{O}_{U}$ be the sheaf of holomorphic functions on $U$ and $\mathcal{I}$ the ideal sheaf in $\mathcal{O}$ generated by $h_{j} u_{i}, 1 \leq j \leq 2 m, 1 \leq i \leq(n+2 m)$. I defines a coherent sheaf on the Stein manifold $U$ and $H^{1}(U, \mathcal{I})=0$ by Cartan's Theorem B. Thus, for the short exact sequence $0 \rightarrow \mathcal{I} \rightarrow \mathcal{O} \rightarrow \mathcal{O} / \mathcal{I} \rightarrow 0$, we have surjectivity for $H^{0}(U, \mathcal{O}) \longrightarrow H^{0}(U, \mathcal{O} / \mathcal{I})$ in the induced long exact sequence. Since $h_{j} u_{i}$ vanishes to the second order at the point $y$, an element on the stalk $\mathcal{O} / \mathcal{I}$ at $y$ corresponds to an equivalence class of germs of holomorphic functions in $U$, where $g_{1}, g_{2} \in \mathcal{O}_{U ; y}$ are equivalent if and only if $\left.g_{1}\right|_{\bar{V}}=\left.g_{2}\right|_{\bar{V}}$ and $d g_{1}(y)=d g_{2}(y)$. In any sufficiently small open neighborhood $\mathcal{W}_{y}$ of $y$ we can always construct for $1 \leq k \leq 2 m$, a holomorphic function $f_{\mathcal{W}_{y} ; k}$ in $\mathcal{W}_{y}$ vanishing on $\bar{V} \cap \mathcal{W}_{y}$ and $d f_{\mathcal{W}_{y} ; k}(y)=d u_{n+k}(y) . f_{\mathcal{W}_{y} ; k}$ induces a section of $\mathcal{O} / \mathcal{I}$ over $\mathcal{W}_{y}$ which is 0 except at $y$, thus defining a global section $s_{k} \in H^{0}(U, \mathcal{O} / \mathcal{I})$, where $s_{k}$ is taken to be 0 outside $\mathcal{W}_{y}$. Hence, the surjectivity above provides us the function $f_{k}$ on $U$ satisfying the desired properties in the claim.

\section{Total geodesy}

Recall the notation in Proposition 3.4. Let $V$ be the irreducible algebraic subvariety extending the graph of $F$ and $W \subset \mathbb{C}^{n}$ be a proper algebraic subvariety such that if we let $Z=\mathbb{C}^{n} \backslash W$ and $X=V \backslash \pi^{-1}(W)$, then $\pi: X \longrightarrow Z$ is a finite unbranched covering map. We start with a lemma.

Lemma 4.1. If a component function is degenerate everywhere in $\mathbb{B}^{n}$, i.e. the tangent map is not injective anywhere, then it is constant.

Proof. Let $A$ be the component function degenerate everywhere. Consider $A$ as a multi-valued map on $Z$ and let $Y$ be the set of points $\mathbf{z} \in Z$ such that $\|A(\mathbf{z})\|=1$ on some branch. Since the functional equation Eq.(3.2) is satisfied on the whole algebraic subvariety $V$, we see that $Y \subset Z \cap \partial \mathbb{B}^{n}$.

Define $Z^{\prime}=Z \backslash Y$. We first argue that by the degeneracy of $A, Z^{\prime}$ is connected. Suppose on the contrary $Z^{\prime}$ is not connected. Because $Y \subset Z \cap \partial \mathbb{B}^{n}$ and $Y$ is closed in $Z, Z^{\prime}$ is not connected only if $Y=Z \cap \partial \mathbb{B}^{n}$. Hence for every point $\mathbf{z}_{\mathbf{0}} \in Z \cap \partial \mathbb{B}^{n}$, there is some branch of 
$A$ on which we have $A\left(\mathbf{z}_{\mathbf{0}}\right)=\mathbf{a}_{\mathbf{0}}$ with $\left\|\mathbf{a}_{\mathbf{0}}\right\|=1$. Because $A$ is degenerate everywhere, for a generic choice of $\mathbf{z}_{\mathbf{0}}$, the set defined by $A(\mathbf{z})=\mathbf{a}_{\mathbf{0}}$ contains a non-constant complex analytic curve $\Gamma: \Delta \longrightarrow \mathbb{C}^{n}$ with $\Gamma(0)=\mathbf{z}_{0}$. Note that for all open set $U \subset \Delta, \Gamma(U)$ cannot be completely contained in $\partial \mathbb{B}^{n}$ and from the functional equation we see that $\Gamma(U) \backslash \partial \mathbb{B}^{n}$ must be contained in $W$. This is true for arbitrary $U$ and this implies that $\mathbf{z}_{0}=\Gamma(0) \in W$. So $W$ contains almost every point of $\partial \mathbb{B}^{n}$ and hence the whole $\partial \mathbb{B}^{n}$ which is not possible.

We now show that the connectedness of $Z^{\prime}$ implies that $A$ is constant. It is clear that $\pi^{-1}\left(Z^{\prime}\right) \subset X$ can only have a finite number of connected components, therefore each connected component is open in $X$ and when $\pi$ is restricted to any one connected component it is still a covering map over $Z^{\prime}$. Since $Z^{\prime}$ is connected, on each connected component we have either $\|A\|<1$ or $\|A\|>1$ on the whole component. We choose one with $\|A\|<1$, of which the existence is guaranteed because we started with an isometric embedding germ $F$ of $\mathbb{B}^{n}$ into a product of unit balls. We can then form elementary symmetric functions of $A$ with respect to this covering map and they are bounded holomorphic functions on $Z^{\prime}$. Since $W$ is a proper subvariety, we can extend them separately throughout the two domains $\mathbb{B}^{n}$ and $\mathbb{C}^{n} \backslash \overline{\mathbb{B}^{n}}$. As $n \geq 2$, the symmetric functions in $\mathbb{C}^{n} \backslash \overline{\mathbb{B}^{n}}$ can be extended to the whole $\mathbb{C}^{n}$ by Hartog's extension and the extension must agree with the symmetric functions originally defined on $\mathbb{B}^{n}$ as $Z^{\prime}$ is connected. Hence, the symmetric functions are bounded holomorphic functions on $\mathbf{C}^{n}$ and therefore constant. This implies that $A$ is constant.

We can now prove the main theorem of this article.

Proof. (of the Main Theorem)

As explained after Proposition 3.2, it remains to prove the total geodesy of a holomorphic isometric embedding $F: \mathbb{B}^{n} \longrightarrow \mathbb{B}^{m} \times \mathbb{B}^{m}, F(\mathbf{z})=(A(\mathbf{z}), B(\mathbf{z}))$ with the isometric constant $\lambda=(m+1) /(n+1)$.

If $m<n$, we certainly have degeneracy for both component functions and by Lemma 4.1 they are constant which is impossible. Therefore $m \geq n$.

By reducing the dimension of the target, we can always assume that the image of one of the component functions, say $B$, does not lie in any proper linear subspace of $\mathbb{B}^{m}$. If the other component $A$ is degenerate everywhere, then $A$ is constant by Lemma 4.1 and hence $A(\mathbf{z}) \equiv \mathbf{0}$. Therefore, up to unitary transformations, we have $F(\mathbf{z})=\left(\mathbf{0}, I_{n ; m}(\mathbf{z})\right)$, where $I_{n ; m}: \mathbb{C}^{n} \longrightarrow \mathbb{C}^{m}$ is the canonical embedding.

Now suppose $F$ is a holomorphic isometric embedding of $\mathbb{B}^{n}$ into $\mathbb{B}^{m} \times \mathbb{B}^{m}$ with $2 n>m \geq$ $n$, such that $A$ is non-degenerate at a generic point and the image of $B$ does not lie in any proper linear subspace of $\mathbb{C}^{m}$. We are going to show that it will lead to a contradiction.

Since the image $B$ do not lie in any proper linear subspace, in particular, it is non-constant and is non-degenerate at a generic point by Lemma 4.1. Therefore we may assume that both $A$ and $B$ are non-degenerate at the origin.

Denote the elements of the unitary matrix $\mathbf{U}$ in Eq.(3.3) by $u_{r s}, 1 \leq r, s \leq\left(m^{2}+n\right)$. Since $a_{i}(0)=b_{j}(0)=0, \forall i, j$ by assumption, if we consider the power series expansions of the last $\left(m^{2}+n-2 m\right)$ equations in Eq.(3.3), we see that $u_{r s}=0$ for $(2 m+1) \leq r \leq\left(m^{2}+n\right)$ and $\left(m^{2}+1\right) \leq s \leq\left(m^{2}+n\right)$. Hence, if we let

$$
\mathcal{X}=\left(a_{1} b_{1}, \ldots, a_{1} b_{m}, \ldots, a_{m} b_{1}, \ldots, a_{m} b_{m}\right)=\left(a_{1} B, \ldots, a_{m} B\right)
$$


be a $\mathbb{C}^{m^{2}}$-valued vector function, then the last $\left(m^{2}+n-2 m\right)$ equations in Eq.(3.3) amounts to saying that there exist $\left(m^{2}+n-2 m\right)$ constant orthonormal vectors $\left\{\mathcal{U}_{j} \in \mathbb{C}^{m^{2}}: 1 \leq j \leq\right.$ $\left.\left(m^{2}+n-2 m\right)\right\}$ such that

$$
\mathcal{X} \perp \operatorname{Span}\left\{\mathcal{U}_{j}\right\}
$$

If we let $X=\operatorname{Span}\left\{\mathcal{U}_{j}\right\}^{\perp}$, then $\operatorname{Dim}(X)=m^{2}-\left(m^{2}+n-2 m\right)=(2 m-n)$ and $\forall \mathbf{z} \in \mathbb{B}^{n}$, $\mathcal{X}(\mathbf{z}) \in X$.

Let $\mathbf{u}$ be a vector in $\mathbb{C}^{n}$. If $\mathbf{u}=\left(u_{1}, \ldots, u_{n}\right)$, define the first directional derivative of a function $g$ along $\mathbf{u}$ as $\frac{\partial g}{\partial \mathbf{u}}:=\sum_{i=1}^{n} u_{i} \frac{\partial g}{\partial z_{i}}$ and second directional derivative along $\mathbf{u}$ as $\frac{\partial^{2} g}{\partial \mathbf{u}^{2}}:=$ $\frac{\partial}{\partial \mathbf{u}} \frac{\partial g}{\partial \mathbf{u}}$

Now, the second directional derivative of $\mathcal{X}$ along $\mathbf{u}$ at $\mathbf{0}$ is

$$
\frac{\partial^{2} \mathcal{X}}{\partial \mathbf{u}^{2}}(\mathbf{0})=\left.\left(2 \frac{\partial a_{1}}{\partial \mathbf{u}} \frac{\partial B}{\partial \mathbf{u}}, \ldots, 2 \frac{\partial a_{m}}{\partial \mathbf{u}} \frac{\partial B}{\partial \mathbf{u}}\right)\right|_{\mathbf{z}=\mathbf{0}} .
$$

By doing unitary transformations in the target, we can assume that the tangent space of the image of $A$ at the origin of $\mathbb{C}^{m}$ is the linear subspace defined by $z_{n+1}=z_{n+2}=\cdots=$ $z_{m}=0$. Therefore we can find $n$ tangent vectors $\mathbf{u}_{\mathbf{1}}, \ldots, \mathbf{u}_{\mathbf{n}}$ such that

$$
\left.\left(\frac{\partial a_{1}}{\partial \mathbf{u}_{i}}, \ldots, \frac{\partial a_{m}}{\partial \mathbf{u}_{i}}\right)\right|_{\mathbf{z}=\mathbf{0}}=E_{i}, \quad 1 \leq i \leq n,
$$

where $E_{i}$ are the standard unit vectors in $\mathbb{C}^{m}$. Then

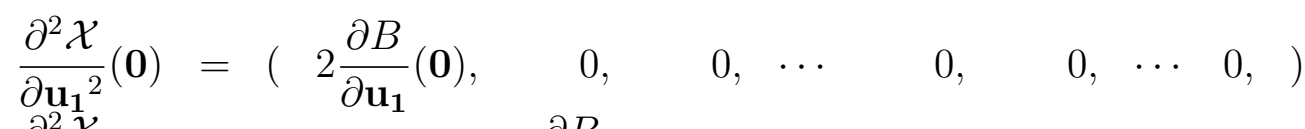

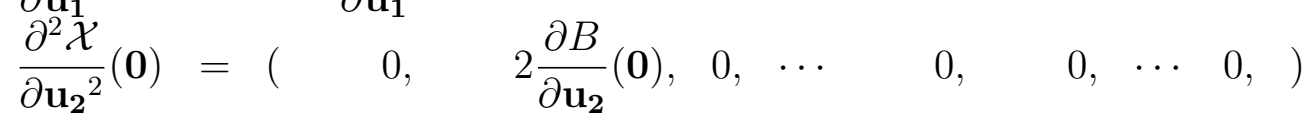

$$
\begin{aligned}
& \frac{\partial^{2} \dot{\mathcal{X}}}{\partial \mathbf{u}_{\mathbf{n}}{ }^{2}}(\mathbf{0})=\left(\begin{array}{llllllll}
0, & 0, & 0, & \cdots & 2 \frac{\partial B}{\partial \mathbf{u}_{\mathbf{n}}}(\mathbf{0}), & 0, & \cdots & 0,
\end{array}\right)
\end{aligned}
$$

Note that for all $i, \frac{\partial^{2} \mathcal{X}}{\partial \mathbf{u}_{i}^{2}}(\mathbf{0}) \in X$. They are linearly independent because $\forall i \frac{\partial B}{\partial \mathbf{u}_{i}} \neq 0$ for $B$ is non-degenerate at the origin. Since $\operatorname{Dim}(X)=(2 m-n)$, we can complete $\left\{\frac{\partial^{2} \mathcal{X}}{\partial \mathbf{u}_{1}^{2}}(\mathbf{0}), \ldots, \frac{\partial^{2} \mathcal{X}}{\partial \mathbf{u}_{n}^{2}}(\mathbf{0})\right\}$ to a basis of $X$ by adding certain $(2 m-2 n)$ vectors in $\mathbb{C}^{m^{2}}$, denoted by $\left\{\mathcal{P}_{1}, \ldots, \mathcal{P}_{2 m-2 n}\right\}$. For each $j$, write $\mathcal{P}_{j}=\left(P_{j}^{1}, \ldots, P_{j}^{m}\right)$, where $P_{j}^{i} \in \mathbb{C}^{m}$.

Since $\mathcal{X}(\mathbf{z}) \in X=\operatorname{Span}\left\{\frac{\partial^{2} \mathcal{X}}{\partial \mathbf{u}_{1}^{2}}(\mathbf{0}), \ldots, \frac{\partial^{2} \mathcal{X}}{\partial \mathbf{u}_{n}^{2}}(\mathbf{0}), \mathcal{P}_{1}, \ldots, \mathcal{P}_{2 m-2 n}\right\}$, by Eq.(4.1) and Eq. (4.2), we see from considering the last $m$ coordinates that for $m=n, B(\mathbf{z}) \in \operatorname{Span}\left\{\frac{\partial B}{\partial \mathbf{u}_{n}}(\mathbf{0})\right\}$ and for $m>n, B(\mathbf{z}) \in \operatorname{Span}\left\{P_{1}^{m}, \ldots, P_{2 m-2 n}^{m}\right\}$. In the first case $(m=n)$, the image of $B$ lies in a subspace of dimension 1 while in the second case $(m>n)$ in a subspace of dimension $2 m-2 n$ which is less than $m$ because $m<2 n$ and therefore in both cases the image of $B$ lies in a proper linear subspace of $\mathbb{C}^{m}$ and this contradicts our initial assumption and the proof is complete. 
Acknowledgement: This article is part of the author's thesis at the University of Hong Kong and he would like to express the gratitude to his supervisor Professor Ngaiming Mok for his inspiring guidance and encouragement.

\section{References}

[1] Clozel L. and Ullmo E. Correspondances modulaires et mesures invariantes. J. Reine Angew. Math., 558:47-83, 2003.

[2] Mok N. Uniqueness of theorems of hermitian metrics of seminegative curvature on locally symmetric spaces of negative ricci curvature. Ann. Math., 125:105-152, 1987.

[3] Mok N. Metric Rigidity Theorems on Hermitian Locally Symmetric Manifolds. Series in Pure Mathematics Vol. 6, World Scientific, Singapore-New Jersey-London-Hong Kong, 1989.

[4] Mok N. Local holomorphic isometric embeddings arising from correspondences in the rank-1 case. In Chern S.S., Fu L., and Hain R., editors, Contemporary Trends in Algebraic Geometry and Algebraic Topology, pages 155-166. Nankai Tracts in Mathematics, Vol 5, World Scientific, New Jersey 2002.

[5] Mok N. Extension of germs of holomorphic isometries up to normalization constants with respect to the Bergman metric. Preprint: http://hkumath.hku.hk/ imr/IMRPreprintSeries/2009/IMR2009-9.pdf. 